\title{
Myocardial Viability Assessment by Dobutamine Echocardiography using Tissue Doppler and Speckle-Tracking: Comparison with SPECT
}

\author{
Attia M. Shokr *, Aly M. Abd Almageed, Abd Elrahman A. Sharaf, \\ Mansour M. Mostafa, Samy H. Nouh, Ahmed M. Fahmy, Mohammad Abdel Hady, \\ Ashraf A. abdelfattah, Mohammad I. Al-Deftar \\ Cardiology department, Faculty of Medicine, Al-Azhar University, Cairo, Egypt \\ attiamorsy2013@yahoo.com
}

\begin{abstract}
:
Background: The use of myocardial strain imaging (MSI) during dobutamine echocardiography (DbE) may facilitate the prediction of myocardial viability. Both tissue-Doppler imaging (TDI) and speckle-tracking echocardiography (STE) are used for myocardial strain measurement. While STE overcomes technical limitations of TDI, it needs validation for prediction of viability.
\end{abstract}

Aim of the work: To determine the relative accuracy of TDI-based and STE-based measurements of myocardial strain and strain rate for the detection of myocardial viability before revascularization using SPECT imaging as agold standard.

Methods: The study included 50 patients (34 males, 16 females; mean age $52 \pm 8$ years) with ischemic heart disease and left ventricular systolic dysfunction; who were undergoing DbE and SPECT after nitrat potentiation for assessment of myocardial viability The patients were divided into two groups according to the result of SPECT study after nitrate potentiation into viable and non-viable myocardial segments for possibility of myocardial revascularization. TDI and STE were used to measure longitudinal peak systolic strain (longS) and peak systolic strain rate (SR) at rest and at low-dose dobutamine (LDD).

Results: Of the 850 segments, 415 segments were non-viable (48.2\%) and 435 segments were viable (51.8\%) according to SPECT with nitrate potentiation, non-viable myocardial segments had significantly lower peak systolic strain and peak longitudinal systolic strain rateat LDD by TDI (areas under the receiver operator characteristic curves of $0.75,0.85$ respectively). Non viable myocardial segments had significantly lower peak systolic strain and peak longitudinal systolic strain rate at LDD by STE (areas under the receiver operator characteristic curves of 0.90, 0.98 respectively).

Conclusion: Strain and strain rate measurements with both TDI and STE at LDD echocardiography can predict myocardial viability. However, STE is more accurate than TDI when both are compared to SPECT results.

Keywords: Myocardial viability; Tissue Doppler Imaging; Speckle tracking Echocardiography; SPECT

\section{INTRODUCTION}

The detection of myocardial viability in patients with ischemic left ventricular (LV) dysfunction has potential therapeutic and prognostic implications [1].

Dobutamine echocardiography (DbE) has comparable accuracy to other approaches for the assessment of myocardial viability (Bax et al., 1977). But involves subjective assessment of wallthickening responses to dobutamine stimulation and is highly operator dependent [2].

Measurement of myocardial deformation with tissue Doppler imaging (TDI) during DbE has comparable accuracy to expert wall-motion analysis for prediction of functional recovery [3].

However, the wider application of Doppler strain in clinical practice has been constrained by its susceptibility to signal noise and dependence on the angle of insonation [4].

Speckle tracking echocardiography (STE) is a new noninvasive ultrasound imaging technique that allows for an objective and quantitative evaluation of global and regional myocardial function independently from the angle of insonation and from cardiac translational movements [5]. 
Recently, speckle-tracking analysis with automated function imaging (AFI) has been introduced as a new echocardiographic technique to assess global LV longitudinal strain [6].

Importantly, speckle-tracking analysis derived strain has been associated with the extent of viable myocardial tissue in patients with chronic ischemic heart disease [7]. Angle dependency is not a problem with 2-dimensional (STE), but this technique is dependent on image quality and operates at limited frame rate [8].

STE-based myocardial strain has been validated against other techniques, used to measure the amplitude and timing of function at rest, and applied (with more difficulty) during stress, However, its utility in the dobutamine echocardiography assessment of myocardial viability has not been studied [9].

\section{Patients AND Methods}

\subsection{Study Population}

Informed consents were obtained from all patients and the study protocol was approved by the local ethics committee of our hospital. The study included 50 subjects, presented to our Cardiology Department for assessment of myocardial viability. According to SPECT after nitrate potentiation findings, myocardial segments were divided into two groups (viable and non-viable myocardial segments).

\subsection{Exclusion Criteria}

Patients with Significant valvular heart disease; Contraindication to dobutamine (including: Unstable patients such as those with decompensated heart failure or unstable angina, aortic stenosis, hypertrophic cardiomyopathy, arrhythmias that interfere with interpretation of dobutamine stress echocardiography or perfusion scintigraphy); Refusal of the to participate in the study; Patients with bad echo window or when echo study protocol cannot be completed.

\subsection{Clinical Evaluation}

A detailed medical history was obtained from every subject and risk factors of CAD were established. Subjects were also evaluated by physical examination and 12-lead electrocardiography. Hypertension, diabetes mellitus were defined by the relevant treatment. Obesity was defined as body mass index (BMI) of $>30 \mathrm{~kg} / \mathrm{m}^{2}$.

\subsection{Conventional Transthoracic Echocardiographic Assessment}

Echo Doppler examination was performed with a Philips iE33 X-Matrix system equipped with TDI \& STE technology using a multi-frequency $(1-5 \mathrm{MHz})$ X5-1 matrix array probe. All the patients were examined in the left lateral decubitus position and echocardiographic images were acquired from the standard views (parasternal long-axis, parasternal short axis at papillary muscle level, apical 4chamber, apical 5-chamber and apical 2-chamber). Recordings and calculations of different cardiac chambers and ejection fractions were made according to the recommendations of the American Society of Echocardiography [10].

\subsection{Low Dose Dobutamine Echocardiography}

Beta-blockers, calcium antagonists and nitrates were discontinued in patients at least 48 hours before low dose dobutamine echocardiography. Low dose dobutamine (LDD) infusion was administered using automated infusion pump. Dobutamine was delivered intravenously using 3 minutes staged protocol starting from $5 \mu \mathrm{g} / \mathrm{kg} / \mathrm{min}$ for three minutes, then $10 \mu \mathrm{g} / \mathrm{kg} / \mathrm{min}$ for another three minutes period, then 3 minutes recovery without dobutamine. Images were acquired from apical four, two \& three chamber views, with superimposition of color tissue Doppler imaging (TDI) and speckle tacking data at the $2 \mathrm{D}$ images.

The recorded 2D image loops were digitally stored at rest and at $10 \mu \mathrm{g} / \mathrm{kg} / \mathrm{min}$ LDD echocardiography for later offline analysis. Patients were continuously monitored by ECG and blood pressure measurement during LDD test.

Dobutamine infusion was inteded to be terminated if one of the following had occurred:

- Severe chest pain or intolerable side effects.

- ST-segment elevation $>1 \mathrm{~mm}$ in leads without a $\mathrm{Q}$ wave. 
- Horizontal ST-segment depression $>2 \mathrm{~mm}$ in any lead.

- Significant ventricular or supraventricular arrhythmias.

- Uncontrolled systemic hypertension $\geq 180 / 110 \mathrm{mmHg}$.

\subsection{Tissue Doppler Imaging (TDI)}

Tissue Doppler strain and strain rate values were obtained from apical views (apical 4-chamber, apical 2-chamber and apical long axis views) at rest \& LDD with measurement peak systolic Strain (calculated as \%) and strain rate values at twelve LV segments of every patient by excluding 4 apical segments from the 16 segment of American society of Echocardiography), the apical segments were excluded because strain TDI data is very angle dependant that limit its use in these segments.

After that we compare the mid and basal segments of posterior septum ,lateral wall ,inferior wall ,anterior wall, posterior wall and anterior septum with results of MPI. The apical parts were excluded because strain TDI data is very angle dependant and Because of the greater angle between the ultrasonic beam and LV axis for apical segments, determination of strain TDI data for apical Segments is likely to be less accurate [11].

\subsection{2-D Speckle Tracking Echocardiography Study}

The following views were taken for later analysis; apical 4 chamber view, apical 2 chamber view, apical long axis view.

In blinded post-processing, longitudinal deformation had been assessed by speckle tracking, being measured the peak systolic longitudinal strain and strain rate for the 17 segment LV model from the apical 4-chambers, 2-chambers and long axis views, with high frame rates (> 60 frames/s). Endsystole was defined as aortic valve closure in the apical long-axis view by continuous Doppler wave recording.Automated delineation of endocardial borders was obtained through marking the mitral annulus level and at the apex on each digital loop. The area of interest was manually adjusted if automated delineation was not optimal. Segments with poor image acquisition or artifacts were excluded due to inability to measure longitudinal strain. Segmental longitudinal strain was calculated as the percentage of lengthening or shortening and the results for each plane were displayed [12].

\subsection{Single Photon Emission Computed Tomography (SPECT) Imaging}

The entire 50 patients underwent myocardial single photon emission computed tomography using Tc99m Sestamibi (Tc-99m-sestaMIBI SPECT). The studies were done in two settings one at rest and the second at rest with nitrate potentiation was done in the next day, Beta-blockers, calcium antagonists and nitrates were discontinued in patients at least 24 hours before the study. After overnight fast, patients underwent qualitative and semi quantitative SPECT, following intravenous administration of 20-25 mCi Tc-99m Sestamibi under resting condition. In the next day the patient was given sublingual isosorbide dinitrate $5 \mathrm{mg}$ after measuring the blood pressure and counting the heart rate and following up the patient for an increase in heart rate to $10 \mathrm{~b} / \mathrm{m}$ over the basal heart rate and/or decrease of blood pressure $10 \mathrm{mmHg}$ of the basal blood pressure measurement. Another tablet of isosorbide dinitrate was given if neither of these happened. Increase of heart rate or drop of blood pressure was the indication for injection of Tc-99m Sestamibi. Patients with blood pressure $\leq 100 / 70$ $\mathrm{mmHg}$, either as baseline blood pressure or in response to Rest-rest with nitrate potentiation protocol was used. To minimize gall bladder activity, the patients were instructed to consume a fatty meal after Sestamibi injection. Computed tomography images were acquired one hour after infusion of the radiotracer. Image acquisition was achieved with a dual head gamma camera (Siemens) without attenuation or scatter correction, using a low energy, all-purpose collimator. Transaxial tomograms were reconstructed for each patient, at short-axis, horizontal and vertical long axis slices and were analyzed. A total of 17 myocardial segments per patient were studied

\subsection{Analysis of Rest- Rest with Nitrate Potentiation Tc-99m-Sestamibi SPECT Images}

All images were reviewed by two experts; both were unaware of the other reading results by qualitative and semi-qualitative analysis. Topographic images were collected from three planes:

Short axis slices from apex to base of the heart were divided into four apical segments (apical anterior, apical septal, apical inferior and apical lateral), six mid-ventricular segments (mid anterior, mid antero-septal, mid infero-septal, mid inferior, mid infero-lateral and mid antero-lateral), and six 
Attia M. Shokr et al.

basal segments (basal anterior, basal antero-septal, basal infero-septal, basal inferior, basal inferolateral and basal antero-lateral). The segments were numbered successively in anti-clockwise manner (see figure 2).Vertical long axis slices from septum to lateral wall. The apical portion of the slice midway between the septum and lateral wall represents the apical segment resulting in a total 17 segments for every patient (16 from short axis and 1 from the vertical long axis views) without duplication of the same anatomic area on different projection [13].Each segment of tomographic images was visually graded as viable if uptake $>40 \%$ or scarred (non-viable) if uptake $<40 \%$.

\section{Results}

This study included 50 consecutive patients with a mean age of $52.7 \pm 8.3$ years, presented to our Cardiology Department at Al-Azhar University Hospital - Cairo - Egypt, in the period from May 2013 to November 2014 for assessment of myocardial viability.

Table(1). General characteristics of the patient population

\begin{tabular}{|l|l|c|c|}
\hline \multicolumn{2}{|c}{ Variable } & \multicolumn{2}{c|}{ Descriptive data } \\
\hline \multirow{2}{*}{ Sex } & Male & $\begin{array}{c}\text { Frequency } \\
\text { (No. = 50) }\end{array}$ & $\begin{array}{c}\text { Percentage } \\
\text { \% }\end{array}$ \\
\cline { 2 - 4 } & Female & 34 & 68 \\
\hline \multirow{2}{*}{ Hypertension } & Negative & 16 & 32 \\
\cline { 2 - 4 } & Positive & 16 & 32 \\
\hline \multirow{2}{*}{ Daibetes mellitus } & Negative & 34 & 68 \\
\cline { 2 - 4 } & Positive & 26 & 52 \\
\hline \multirow{2}{*}{ Obesity } & Negative & 24 & 48 \\
\cline { 2 - 4 } & Positive & 33 & 66 \\
\hline \multirow{2}{*}{ Smoking } & Negative & 17 & 34 \\
\cline { 2 - 4 } & Positive & 23 & 54 \\
\hline
\end{tabular}

Table(2). Left ventricular dimensions, volumes and function among the study group.

\begin{tabular}{|l|c|c|c|c|c|c|}
\hline \multicolumn{7}{|c|}{ Descriptive Statistics } \\
\hline LVEDD & \multicolumn{7}{|c|}{ Range } & Mean & \pm & SD \\
\hline LVESD & 4.90 & - & 6.80 & 5.73 & \pm & 0.48 \\
\hline LVEDV & 3.00 & - & 4.70 & 3.86 & \pm & 0.44 \\
\hline LVESV & 56.00 & - & 93.00 & 71.16 & \pm & 10.44 \\
\hline EF & 25.00 & - & 64.00 & 43.64 & \pm & 10.83 \\
\hline
\end{tabular}

Table(3). Distribution of viable and non-viable segments (as assessed by SPECT after nitrate potentiation) in the study group.

\begin{tabular}{|c|c|c|c|c|}
\hline \multirow{2}{*}{ Segment } & \multicolumn{2}{|c|}{ Non- viable } & \multicolumn{2}{c|}{ Viable } \\
\cline { 2 - 5 } & No. & No. & \% \\
\hline Basal inferoseptal & 25 & 50 & 25 & 50 \\
\hline Mid inferoseptal & 20 & 40 & 30 & 40 \\
\hline Basal anteroseptal & 30 & 60 & 20 & 40 \\
\hline Mid anteroseptal & 30 & 60 & 20 & 40 \\
\hline Aapicoseptal & 30 & 60 & 20 & 100 \\
\hline Basal anterolateral & 0 & 0 & 50 & 100 \\
\hline Mid anterolateral & 0 & 0 & 50 & 80 \\
\hline Basal inferolateral & 10 & 20 & 40 & 80 \\
\hline Mid inferolateral & 10 & 20 & 40 & 60 \\
\hline Apicolateral & 20 & 40 & 30 & 40 \\
\hline Basal inferior & 30 & 60 & 20 & 40 \\
\hline Mid inferior & 30 & 60 & 20 & 20 \\
\hline Apicoinferior & 40 & 80 & 10 & 40 \\
\hline Basal anterior & 30 & 60 & 20 & 40 \\
\hline Mid anterior & 30 & 60 & 20 & 20 \\
\hline Apicoanterior & 40 & 80 & 10 & 20 \\
\hline Apex & 40 & 80 & 10 & \\
\hline
\end{tabular}


Myocardial Viability Assessment by Dobutamine Echocardiography using Tissue Doppler and SpeckleTracking: Comparison with SPECT

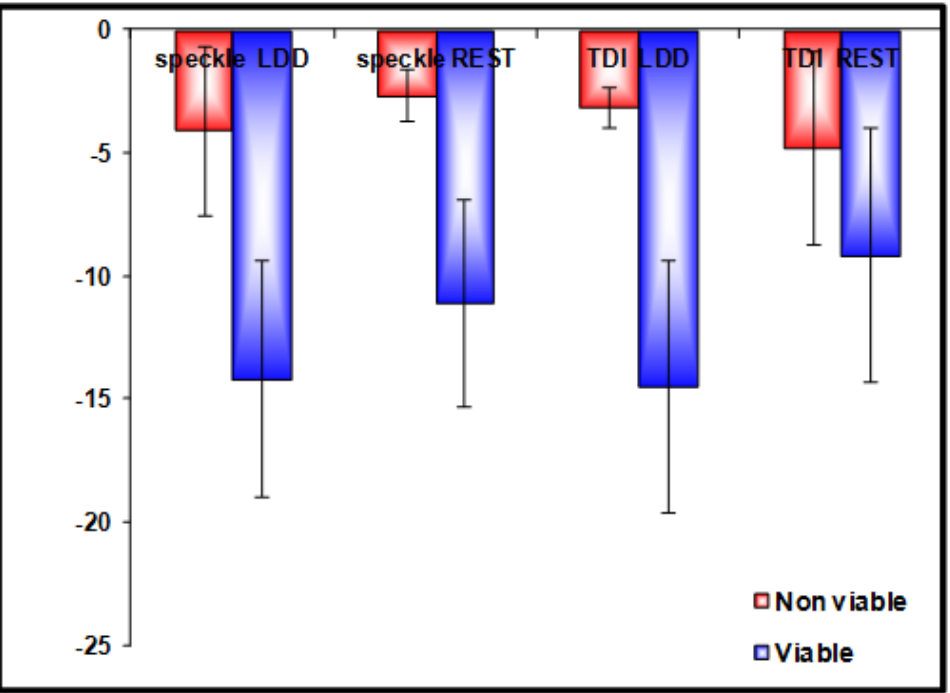

Figure(1). Comparison of peak longitudinal strain (ST) by STE \& TDI at rest and LDD in viable and non-viable myocardial segments.

\begin{tabular}{|c|c|c|c|c|c|c|c|}
\hline \multirow{3}{*}{ LV long. strain (\%) } & \multicolumn{6}{|c|}{ SPECT } & \multirow[b]{3}{*}{ P-value } \\
\hline & \multicolumn{3}{|c|}{ Non viable } & \multicolumn{3}{|c|}{ Viable } & \\
\hline & Mean & \pm & SD & Mean & \pm & SD & \\
\hline STE LDD & -4.07 & \pm & 3.41 & -14.16 & \pm & 4.77 & $<0.001 *$ \\
\hline STE Rest & -2.62 & \pm & 1.02 & -11.06 & \pm & 4.23 & $<0.001 *$ \\
\hline TDI LDD & -3.17 & \pm & 0.82 & -14.45 & \pm & 5.09 & $<0.001 *$ \\
\hline TDI Rest & -4.75 & \pm & 3.95 & -9.12 & \pm & 5.18 & $<0.001 *$ \\
\hline
\end{tabular}

$L V=$ Left Ventricle; STE = Speckle Tracking Echocardiography; TDI = Tissue Doppler Imaging; LDD = Low dose dobutamin; SPECT = single photon emission computed tomography.

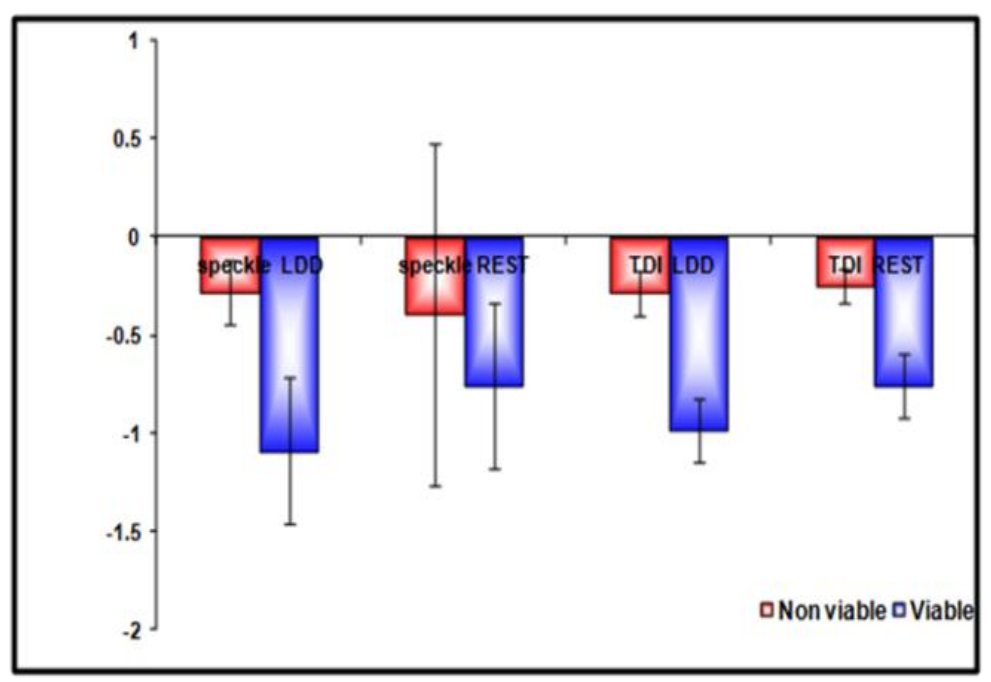

Figure(2). Comparison of longitudinal strain rate (LSR) by STE \& TDI at rest and LDD in viable and nonviable myocardial segments

\begin{tabular}{|c|c|c|c|c|c|c|c|}
\hline \multirow{3}{*}{$\begin{array}{c}\text { Long SR } \\
\left(\mathrm{s}^{-1}\right)\end{array}$} & \multicolumn{6}{|c|}{ SPECT } & \multirow[b]{3}{*}{ P-value } \\
\hline & \multicolumn{3}{|c|}{ Non viable } & \multicolumn{3}{|c|}{ Viable } & \\
\hline & Mean & \pm & SD & Mean & \pm & SD & \\
\hline STE LDD & -0.28 & \pm & 0.16 & -1.09 & \pm & 0.38 & $<0.001^{*}$ \\
\hline STE Rest & -0.40 & \pm & 0.87 & -0.76 & \pm & 0.42 & $<0.001^{*}$ \\
\hline TDI LDD & -0.29 & \pm & 0.11 & -0.99 & \pm & 0.16 & $<0.001^{*}$ \\
\hline TDI Rest & -0.25 & \pm & 0.09 & -0.76 & \pm & 0.16 & $<0.001^{*}$ \\
\hline
\end{tabular}

$L V=$ Left Ventricle; Long SR = Longitudinal Strain rate; STE =M Speckle Tracking Echocardiography; TDI = Tissue Doppler Imaging; $L D D=$ Low dose dobutamin; SPECT = single photon emission computed tomography. 

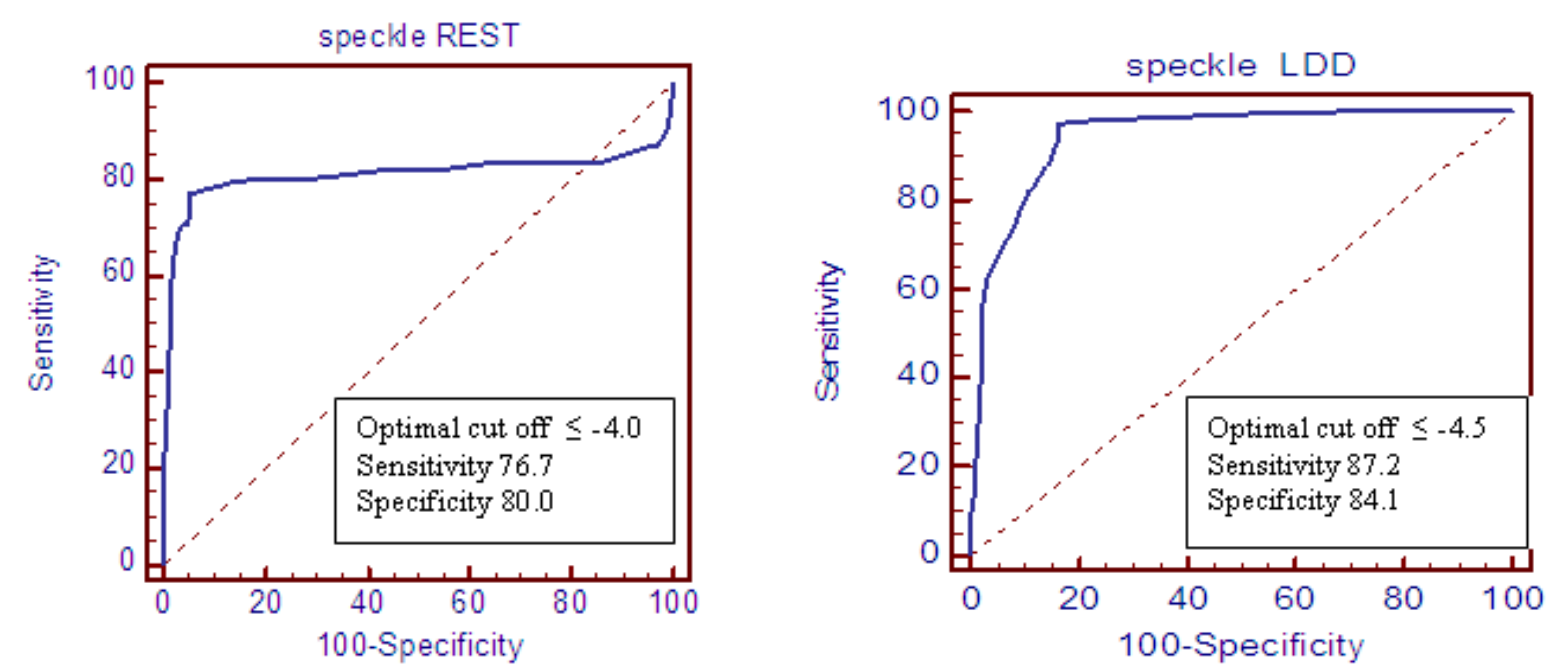

Figure(3). Diagrams showing the ROC curves results for the peak longitudinal systolic strain by speckle tracking at rest (left) and at LDD (right).
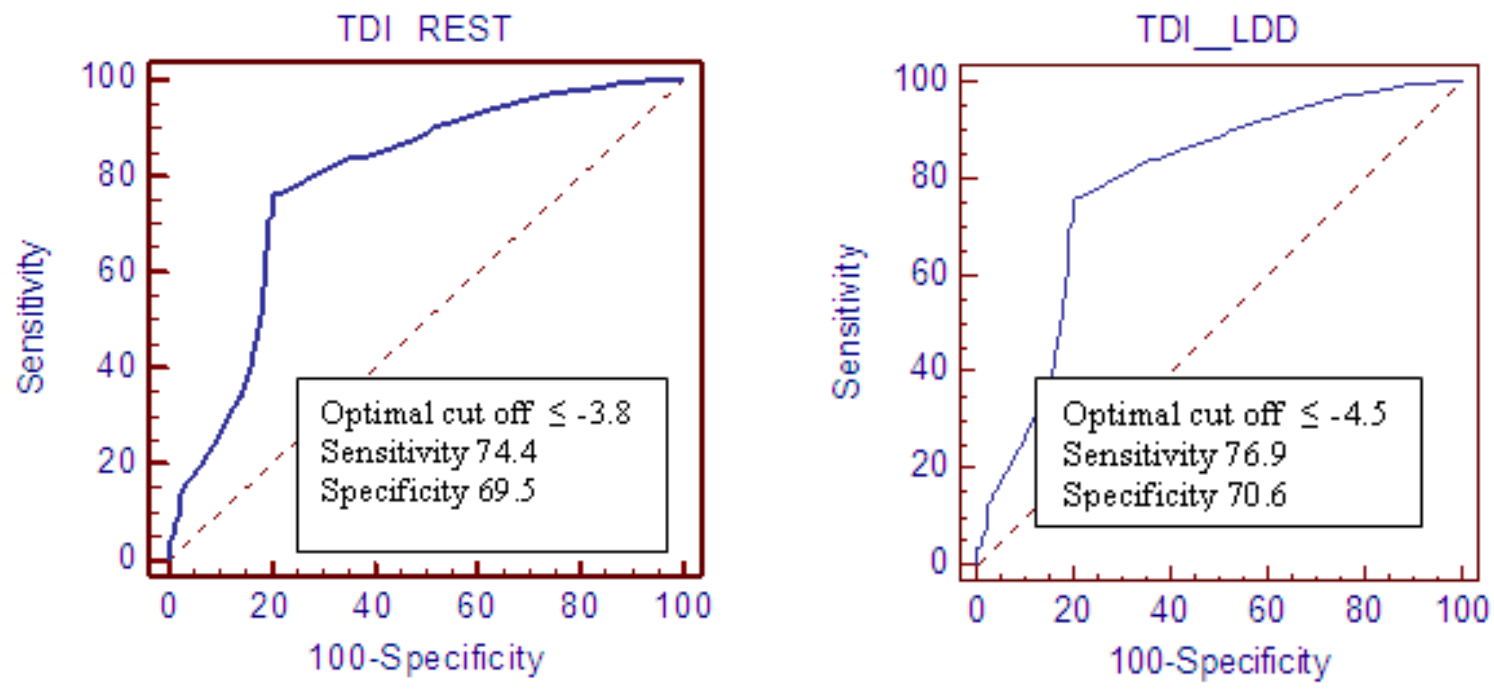

Figure(4). Diagrams showing the ROC curves results for the peak longitudinal systolic strain by TDI at rest (left) and at LDD (right).
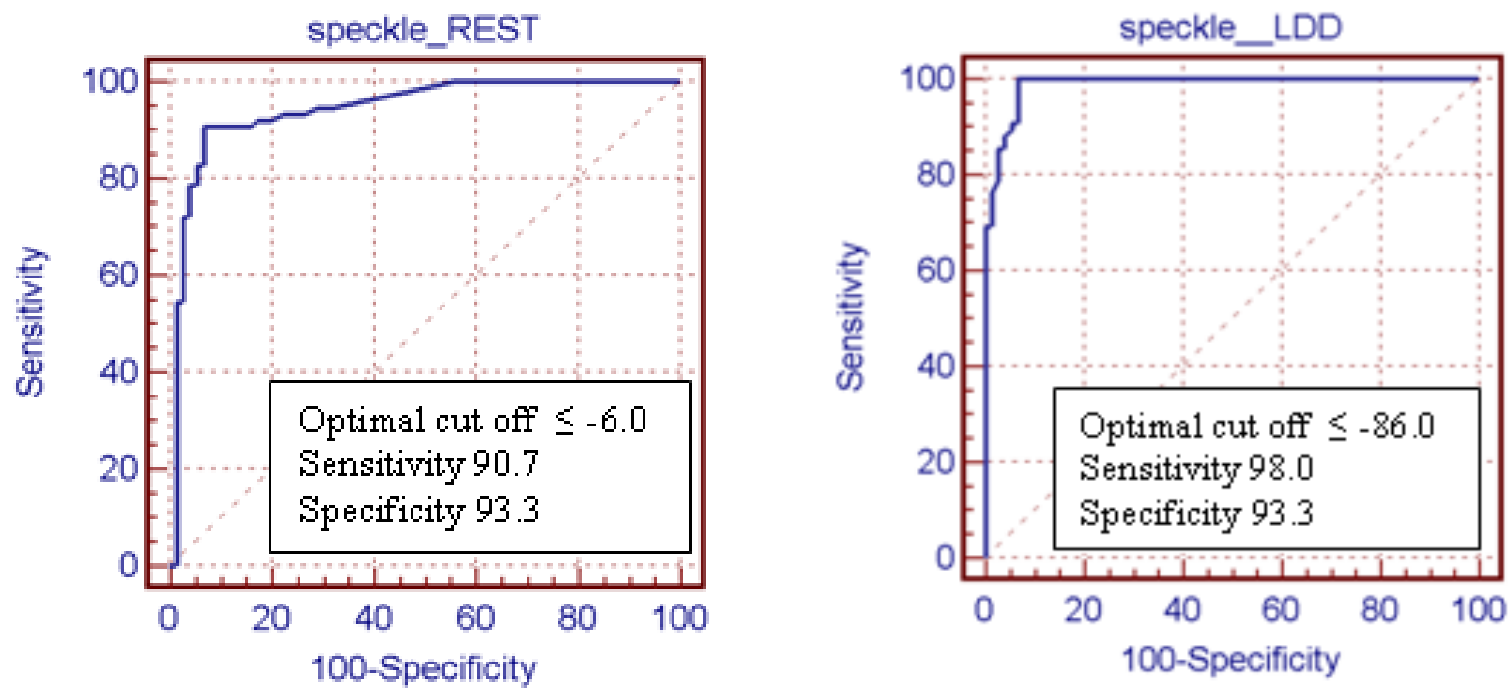

Figure(5). Diagrams showing the ROC curves results for the longitudinal strain rate by speckle tracking at rest (left) and at LDD (right). 
Myocardial Viability Assessment by Dobutamine Echocardiography using Tissue Doppler and SpeckleTracking: Comparison with SPECT
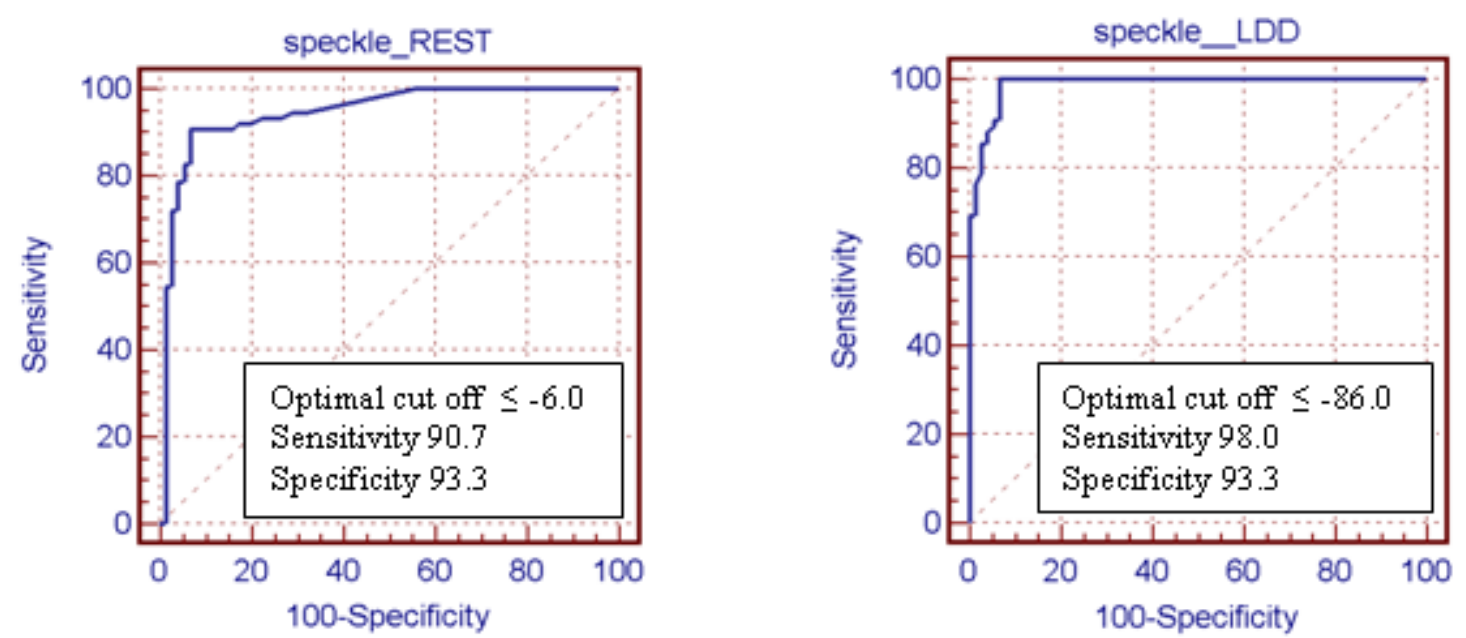

Figure(5). Diagrams showing the ROC curves results for the longitudinal strain rate by speckle tracking at rest (left) and at LDD (right).
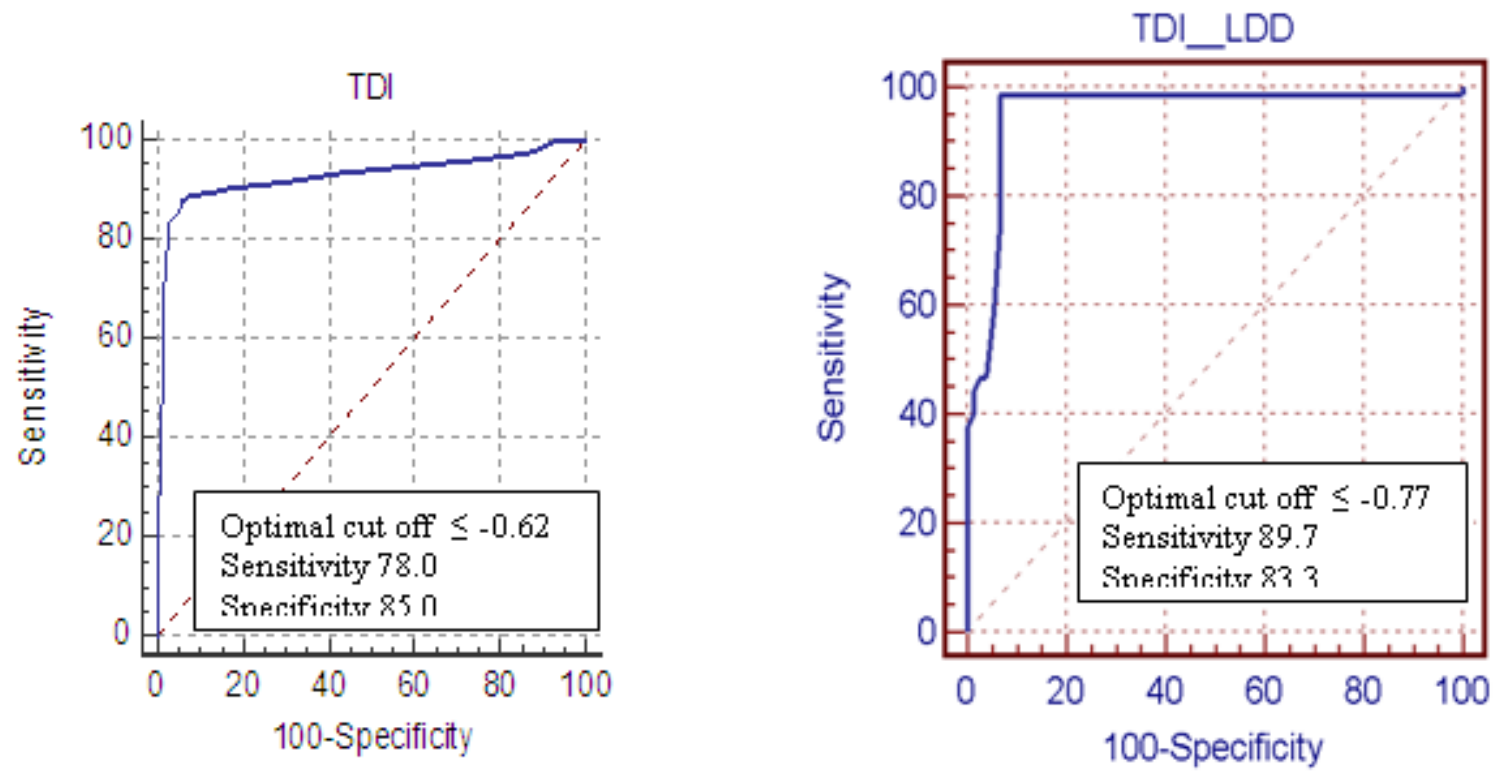

Figure(6). Diagrams showing the ROC curves results for the longitudinal strain rate by TDI at rest (left) and at LDD (right).
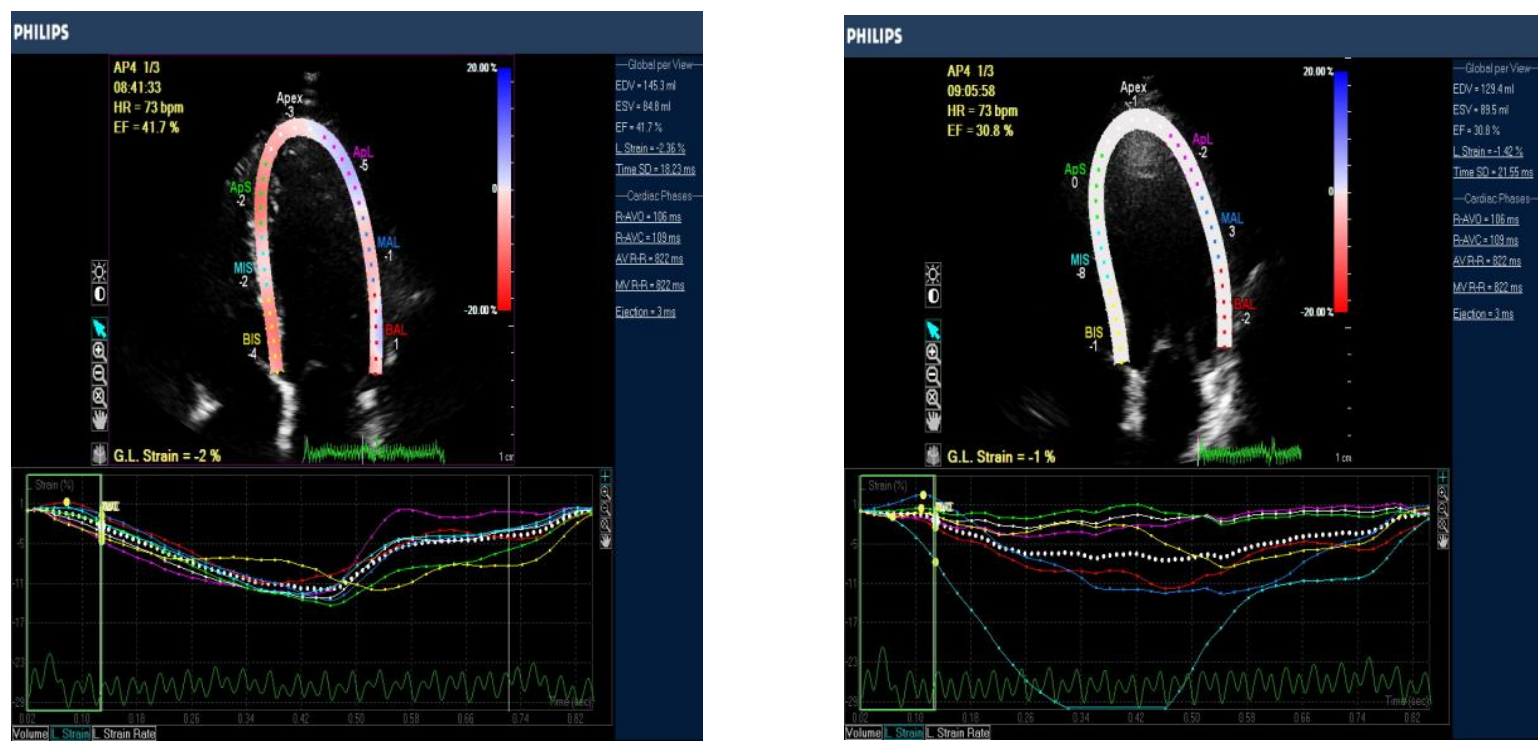

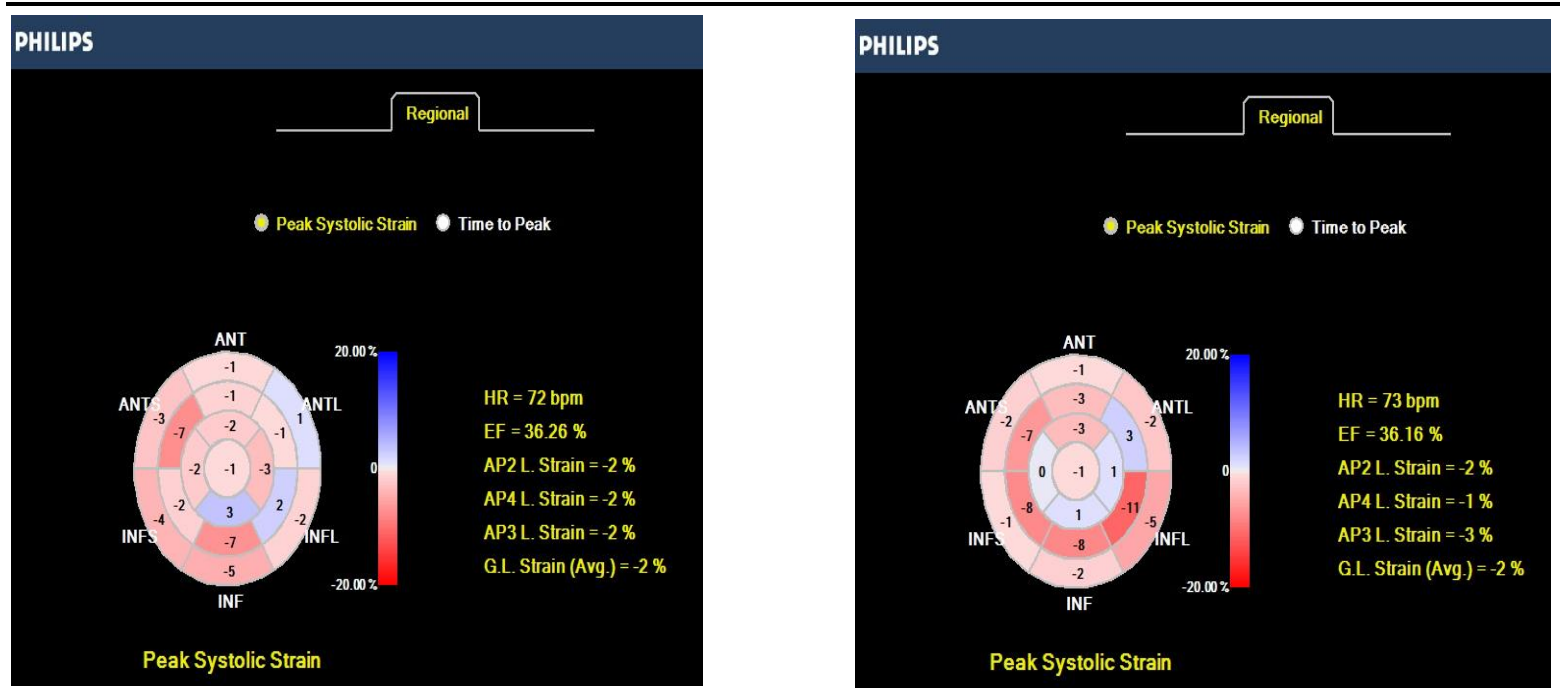

Figure(7). Segmental Peak longitudinal strain by speckle tracking at rest (left) and at LDD (right) in apical 4 chamber view (upper panel) and in Bull's eye map (lower panel) from a male patient having anterior myocardial infarction.

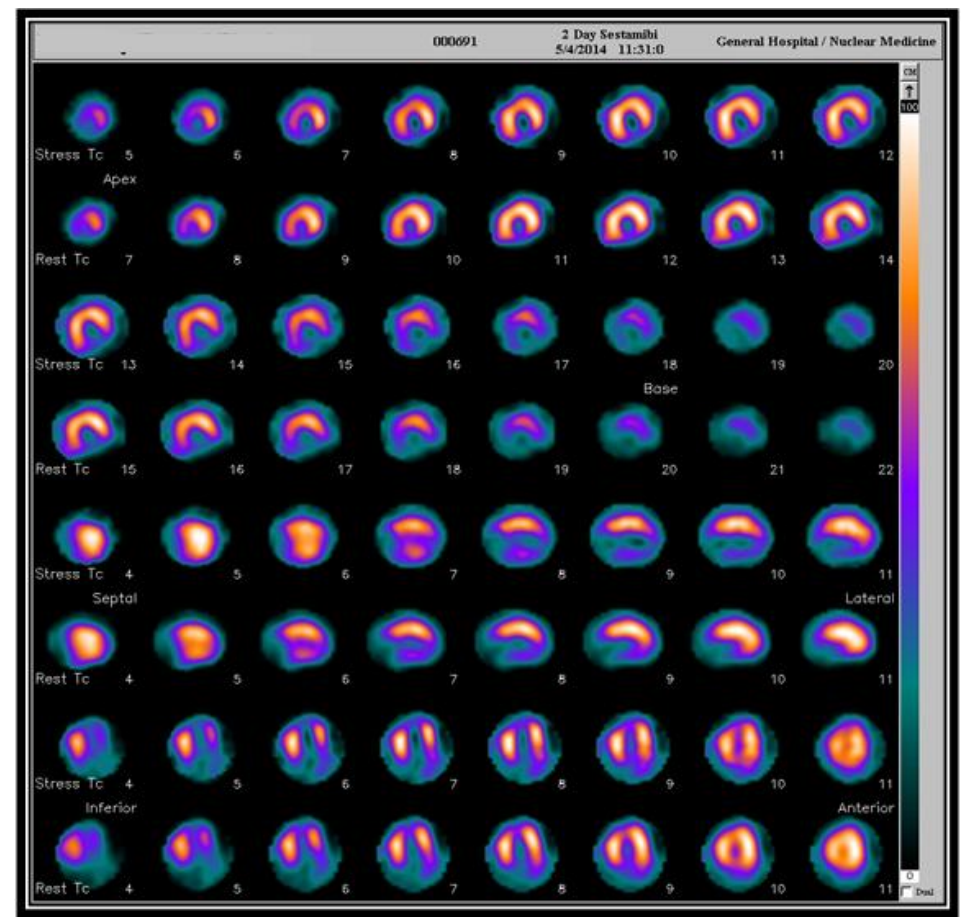

Figure(8). SPECT after nitrate potentiation of the same patient in fig. 7 showing fixed defect at the apex, apicoanterior, inferolateral and inferior segments, combination of scar and ischemia at mid lateral wall.

\section{DISCUSSION}

This study include fifty Patients with ischemic heart disease presented for assessment of myocardial viability for possibility of myocardial revascularization.

Myocardial segments in this study were classified into viable and non-viable groups according to the results of SPECT. Conventional echo measures, TDI \& STE parameters during low dose dobutamine (LDD) echocardiography were evaluated as markers of viability.

In the current study, comparison of echocardiographic parameters in patients with viable versus those with non-viable myocardium, revealed no statistically significant differences between both groups in nearly all conventional echocardiographic measures except for lower LVEF in the non-viable groups compared to the viable group of the corresponding territory.

In the current study, we found lower strain and strain rate values at rest using STE in the non-viable segments compared to the viable groups of the corresponding territory. An increase of strain and strain rate values in response to LDD was detected in the viable group but not in the non-viable ones. 
Regarding strain values, A cut-off point to predict myocardial viability using the ROC curve. At > $4.5 \%$ peak longitudinal systolic strain by STE at LDD chosen as a cut-off point, the positive predictive value for the detection of myocardial viability was $84.55 \%$ and negative predictive value was $96.70 \%$ with a sensitivity of $87.24 \%$ and a specificity of $84.10 \%$.

As regard strain rate values a cut-off point to predict segmental myocardial viability the ROC curve. At $>-0.24 \%$ increasing of segmental longitudinal strain (rate $1 / \mathrm{s})$ at LDD $(10 \mu / \mathrm{kg} / \mathrm{min})$ by STE chosen as a cut-off point, with a sensitivity of $85.29 \%$ and a specificity of $93.73 \%$ and accuracy $88 \%$.

The results of the current study are in agreement with those of Larysa et al,. 2015 [14]. who assess myocardial viability by STE in patients with coronary artery disease and type 2 diabetes mellitus, LV myocardial segments were analyzed by STE before and one year after revascularization, and they found that, patients with coronary artery disease and type 2 diabetes mellitus had lesser degree of LV functional recovery one year after revascularization compared to those without type 2 diabetes mellitus. Assessments of LV myocardial viability using STE is recommended for patients selection before revascularization and further follow up.

Loïc Bière et al., 2014 [15]. were assessed the value of STE performed early after a first ST-segment elevation myocardial infarction in order to predict infarct size and functional recovery at 3-month follow-up. Longitudinal strain $>-6.0 \%$ within the infarcted area exhibited $96 \%$ specificity and $61 \%$ sensitivity for predicting the persistence of akinesia at 3-month follow-up. Speckle-tracking strain imaging performed early after a STEMI is easy-to-use as a marker for persistent akinetic territories at 3 months. and this was compatible to our findings except a cut of point value because they depend on Late gadolinium-enhanced cardiac magnetic imaging as a gold slandered for myocardial viability.

The results of the current study are in agreement with those of Gong L et al., 2013 [16]. who found that Among the radial, circumferential, and longitudinal strain and strain rate parameters, only longitudinal strain and longitudinal strain rate at rest and LDDSE emerged as independent predictors of viable myocardium, When combining longitudinal strain and longitudinal strain rate at LDDSE, the sensitivity, specificity and accuracy for the assessment of viable myocardium rose to 89.8, 90.2 and $89.9 \%$, respectively. The sensitivity of STE in conjunction with LDDSE was similar to dual isotope simultaneous acquisition single photon emission computed tomography (DISA-SPECT) for detecting viable myocardium in patients with AMI, but the specificity and accuracy of STE performed with LDDSE were higher than DISA-SPECT.

Similarly, Martin et al., 2013 [17]. were compare the speckle tracking echocardiography derived systolic longitudinal strain with rest single photon emission computed tomography perfusion imaging and to define the optimal cut-offs for peak longitudinal systolic strain to discriminate transmural scar on contrast-enhanced magnetic resonance imaging (ceCMR). Correlation was found between regional peak longitudinal systolic strain and DE on ceCMR. The peak longitudinal systolic strain optimal cutoff $-5.3 \%$ identified segments with delayed enhancement $>75 \%$ on ceCMR (sensitivity $83.1 \%$, specificity $84.6 \%$ ). STE enabled identification of LV non-viable segments. In comparison with rest myocardial SPECT perfusion imaging, STE is more accurate in predicting non-viable myocardium, and this was compatible to our findings.

Also, Bochenek et al, 2011[18] found that peak systolic longitudinal strain is a powerful predictor of myocardial recovery and this was also compatible with our findings.

The results of the current study are in agreement with those of Roes SD et al, in 2009[19]. who found that a regional longitudinal strain cutoff value of $-4.5 \%$ distinguished nontransmural from transmural infarction with high sensitivity and specificity (81.2\% and $81.6 \%$, respectively).

As regard longitudinal strain rate Zhang et al., in 2005 [20] compared DSI and ceCMR in 47 patients with a first myocardial infarction, An SR $>-0.59 \mathrm{~s}^{-1}$ detected transmural infarction with high sensitivity and specificity (90.9\% and $96.4 \%$, respectively) and a $-0.98 \mathrm{~s}^{-1}>\mathrm{SR}>-1.26 \mathrm{~s}^{-1}$ discriminated subendocardial infarction from normal myocardium with very good sensitivity and specificity $(81.3 \%$ and $83.3 \%$, respectively. and this was also compatible with our findings.

In this study, we found lower strain and strain rate values at rest using TDI in the non-viable segments compared to the viable groups of the corresponding territory. An increase of strain and strain rate values in response to LDD was detected in the viable group but not in the non-viable ones. At > -4.5 
$\%$ peak longitudinal systolic strain by TDI at LDD chosen as a cut-off point to predict myocardial viability using the ROC curve with positive predictive value $78.7 \%$, a sensitivity of $76.94 \%$ and a specificity of $70.76 \%$.

As regard strain rate values a cut-off point At $>-0.77 \%$ peak longitudinal systolic strain rate by TDI at LDD to predict segmental myocardial viability with a sensitivity of $89.7 \%$ and a specificity of $83.3 \%$ and accuracy $85 \%$. Also, an increasing of segmental longitudinal strain rate $\left(\mathrm{s}^{-1}\right)$ at LDD (10 $\mu / \mathrm{kg} / \mathrm{min}$ ) by TDI $>-0.23 \mathrm{~s}^{-1}$ was a good predictor of myocardial viability with a sensitivity of $77.22 \%$ and a specificity of $84.4 \%$ and accuracy $80 \%$.

This results are in agreement with those of Bansal et al., 2010 [21] who found that both longitudinal strain and strain rate at rest and at LDD were significantly higher in segments that recovered after revascularization as compared with those that did not recover but longitudinal strain and strain rate at LDD were stronger predictors of functional recovery than the corresponding parameters at rest. They reported that increment in longitudinal strain (optimal cutoff -3.0 for sensitivity and specificity 0.70 ) and increasing of strain rate (optimal cutoff -0.24 for sensitivity 0.77 and specificity 0.68 ) with dobutamine stimulation were also good predictors of recovery.

On the other hand, Sadeghian et al., 2009 [22] reported reduction in strain and strain rate in akinetic non-viable inferobasal compared to normal segments and the range of strain rate in the studied segments did not overlap with that of normal segments.

The results of the current study are also in agreement with findings of Ohara et al., 2007 [23] who found that end-systolic strain and peak strain were significantly lower in the non-viable group than in the viable group $(-4.8 \pm 4.8 \%$ vs $-9.9 \pm 4.7 \%, \mathrm{p}<0.005 ;-9.9 \pm 4.6$ vs $-13.5 \pm 4.1 \%$, p $<0.05)$. Also, Chan et al., in 2006 [24 ] found that viable myocardium showed higher strain rates than did scar tissue after dobutamine infusion. They reported that augmentation of strain and strain rate with dobutamine is a marker of myocardial viability, which was compatible with our findings.

Also, Jamal et al., 2001[25] reported that, the assessment of myocardial viability based on wall motion scoring during low-dose dobutamine infusion is subjective and often difficult. DSI measures may be useful to identify viable myocardium. Stunned myocardium displays reduced systolic SRs that improve with dobutamine infusion or recovery.

The results of the current study are also in agreement with findings of Hoffmann et al., 2002 [26] who found that an increase in systolic SR of $\geq 0.23 \mathrm{~s}^{-1}$ during low-dose dobutamine infusion had a sensitivity and specificity of $83 \%$ and $84 \%$, respectively, for the detection of viable myocardium when using $18 \mathrm{~F}$-fluorodeoxyglucose cardiac PET as the gold standard.

Also, Hanekom et al., in 2005 [27] reported that the incremental value of strain rate imaging (SRI) parameters over low-dose dobutamine wall motion scoring for predicting functional recovery 9 months after revascularization was studied in 55 patients with a previous myocardial infarction. Abnormal segments that recovered function had lower wall motion score, strain and SRs, with similar sensitivity and specificity for predicting functional recovery; however, the combination of wall motion score and SRI parameters increased the sensitivity and specificity for prediction of functional recovery over wall motion scoring alone, which was compatible with our findings.

\section{LiMitation OF THE STUDY}

This study has some limitations which should be addressed in further studies:

- Relatively small study group.

- Only longitudinal STE parameters analyzed

- A technical limitation is that speckle-tracking echocardiography is dependent on frame rate as well as image resolution. Low frame rate results in the speckle pattern changing too much from frame to frame, which prevents the precise characterization of regional myocardial motion and impacts the overall temporal resolution of the regional strain map. In contrast, increasing the frame rate reduces scan line density which reduces image resolution.

- Tissue Doppler imaging is an angle dependant, therefore the apical segments were excluded because they couldn't be assessed by this technique. 


\section{REFERENCES}

[1] Allman KC, Shaw LJ Hachamovitch R and Udelson JE: Myocardial viability testing and impact of revascularization oprognosis in patients with coronary artery disease and left ventricular dysfunction: a meta-analysis. J Am Coll Cardiol. 2002; 39: 1151-1158.

[2] Picano E, Lattanzi F, Orlandini A, et al. Stress echocardiography and the human factor: the importance of being expert. J Am Coll Cardiol. 1991; 17:666-669,

[3] Hanekom L, Jenkins C, Jeffries L, et al. Incremental value of strain rate analysis as an adjunct to wall-motion scoring for assessment of myocardial viability by dobutamine echocardiography: a follow-up study after revascularization. Circulation. 2005; 112:3892-3900.

[4] Urheim S, Edvardsen T, Torp H, et al. Myocardial strain by Doppler echocardiography: Validation of a new method to quantify regional myocardial function.

[5] Geyer H, Caracciolo G, Abe H. Assessment of myocardial mechanics using speckle tracking echocardiography: fundamentals and clinical applications. J Am Soc Echocardiogr, 2010; 23:351-369.

[6] Belghitia $\mathrm{H}$, Brette $\mathrm{S}$, Lafitte $\mathrm{S}$, et al. Automated function imaging: a new operator- independent strain method for assessing left ventricular function. Arch Cardiovasc Dis. 2008; 101:163-9.

[7] Gjesdal O, Hopp E, Vartdal T, et al. Global longitudinal strain measured by two-dimensional speckle tracking echocardiography is closely related to myocardial infarct size in chronic ischaemic heart disease. Clin Sci (Lond). 2007; 113:287-96.

[8] Langeland S, D'Hooge $\mathrm{J}$,Wouters $\mathrm{PF}$, et al. Experimental validation of a new ultrasound method for the simultaneous assessment of radial and longitudinal myocardial deformation independent of insonation angle. Circulation. 2005; 112:2157-62.

[9] Becker M, Hoffmann R, Kuhl HP, et al. Analysis of myocardial deformation based on ultrasonic pixel tracking to determine transmurality in chronic myocardial infarction. Eur Heart J. 2006; 27:2560-2566.

[10] Lang RM, Bierig M, Devereux RB, et al. Recommendations for chamber quantification: a report from the American Society of Echocardiography's Guidelines and Standards Committee and the Chamber Quantification Writing Group, developed in conjunction with the European Association of Echocardiography, a branch of the European Society of Cardiology. J Am Soc Echocardiogr. 2005; 18: 1440-1463.

[11] Heimdal A, Stoylen A, Torp H, et al. Real time strain rate imaging of the left ventricle by ultrasound. J Am Soc Echocardiogr. 1998; 11: 1013-1019.

[12] Anwar AM. Global and segmental myocardial deformation by 2D speckle tracking compared to visual assessment. World Journal of Cardiology. 2012; 4:341 - 346.

[13] Cerqueira, M.D., Weissman, N.J., Dilsizian, V, et al. Standardized Myocardial Segmentation and Nomenclature for Tomographic Imaging of the Heart.Circulation. 2002; 105: 539-542.

[14] Laryza $Z$ and Natalia L. Myocardial viability assessment by speckle-tracking echocardiography in patients with coronary artery disease and type 2 diabetes mellitus, $J$ Eur Soc Franç Cardiol. 2015; 7: 14-17.

[15] Loïc B, Erwan D, Gwenola T, et al. Longitudinal Strain Is a Marker of Microvascular Obstruction and Infarct Size in Patients with Acute ST-Segment Elevation Myocardial Infarction. J Eur PMC. 2014; 10: 1371.

[16] Gong L, Li D, Chen J, et al. Assessment of myocardial viability in patients with acute myocardial infarction by two-dimensional speckle tracking echocardiography combined with low-dose dobutamine stress echocardiography. Int J of cardiovasc imaging. 2013; 29: 10171028 .

[17] Martin H, Tomas S, Milan K, et al. Speckle tracking echocardiography derived systolic longitudinal strain is better than rest single photon emission tomography perfusion imagingfor nonviable myocardium identification. Biomed Pap Med Fac Univ. 2013; 157:12-21.

[18] Bochenek T, Wita K, Tabor Z, et al. Value of speckle-tracking echocardiography for prediction of left ventricular remodeling in patients with ST-elevation myocardial infarction treated by primary percutaneous intervention. J Am Soc Echocardiogr. 2011; 24(12):1342-8. 
[19] Roes SD, Mollema SA, Lamb HJ, et al.Validation of echocardiographic two-dimensional speckle tracking longitudinal strain imaging for viability assessment in patients with chronic ischemic left ventricular dysfunction and comparison with contrast-enhanced magnetic resonance imaging. Am J Cardiol 2009; 104:312-317.

[20] Zhang Y, Chan AK, Yu CM, et al. Strain rate imaging differentiates transmural from nontransmural myocardial infarction: a validation study using delayed-enhancement magnetic resonance imaging. J Am Coll Cardiol 2005; 46:864-871.

[21] Bansal M1, Jeffriess L, Leano R, et al.Assessment of myocardial viability at dobutamine echocardiography by deformation analysis using tissue velocity and speckle-tracking. J Am Coll Cardiovasc Imaging. 2010; 3(2):121-31.

[22] Sadeghian H, Majd-Ardakani J , Lotfi-Tokaldany M, et al.Comparison between dobutamine stress echocardiography and myocardial perfusion scan to detect viable myocardium in patients with coronary artery disease and low ejection fraction. Hellenic J Cardiol. 2009; 50:45-51.

[23] Ohara Y, Hiasa Y, Hosokawa S, et al. Usefulness of ultrasonic strain measurements to predict regional wall motion recovery in patients with acute myocardial infarction after percutaneous coronary intervention. Am J Cardiol. 2007; 99: 754-759.

[24] Chan J, Hanekom L,Wong C, et al. Differentiation of subendocardial and transmural myocardial infarction using two-dimensional strain rate imaging to assess short and long axis function. $\mathrm{J} \mathrm{Am}$ Coll Cardiol 2006; 48: 2026-2033.

[25] Jamal F, Strotmann J, Weidemann F, et al. Noninvasive quantification of the contractile reserve of stunned myocardium by ultrasonic strain rate and strain. Circulation. 2001; 104:1059- 1065.

[26] Hoffmann R, Altiok E, Nowak B, et al. Strain rate measurement by Doppler echocardiography allows improved assessment of myocardial viability in patients with depressed left ventricular function. J Am Coll Cardiol . 2002; 39:443- 449.

[27] Hanekom L, Jenkins C, Jeffries L, et al. Incremental value of strain rate analysis as an adjunct to wall-motion scoring for assessment of myocardial viability by dobutamine echocardiography: a follow-up study after revascularization. Circulation 2005; 112:3892-3900. 